\title{
FORM FINDING OF THE SCST STRUCTURES BY MEANS OF CABLE TENSIONING
}

\author{
JEUNG-HWAN DOH ${ }^{1}$, JIN-WOO KIM ${ }^{2}$ and MOON-HO PARK ${ }^{3}$ \\ ${ }^{1}$ Griffith School of Engineering, Griffith University, Queensland, Australia. \\ ${ }^{2}$ Department of Civil Engineering, Gyeongsang National University, \\ Gyeongnam, Korea. \\ ${ }^{3}$ Department of Civil Engineering, Kyungpook National University, Daegu, Korea.
}

\begin{abstract}
This paper is focused on the form finding of three kinds of cable-tensioned SCST structures that are constructed with bolt type joint by gusset plate, only bolt type joint, and ball type (Mero type) joint. The cable tensioning method for SCST structure is an innovative, fast, and economical construction method in the special construction site or field condition. In this paper, the unit shape of test models consist of uniform pyramids, from the planar structures on the ground, the space structures are shaped and erected into its final curved space structures by means of cable tensioning. The feasibility of the proposed cable-tensioning technique and the reliability of the established geometric model were confirmed with nonlinear finite element analysis and experiments for a small-scale test models. Through the experiments in the SCST structures by means of cable tensioning, within the yield limit of the members, a planar layout can be deformed to desired space shape. As a results, the proposed cable tensioning technique could be applied the form finding of the various SCST structures, so we can know the behavior characteristic of the joint for each types in practical design purposes. For a special site condition of construction field, this cable tensioning technique should be an economic and reasonable construction method compare to conventional construction method including the heavy crane and scaffold.
\end{abstract}

Keywords: Ball type joint, bolt type joint, cable tensioning, form finding, nonlinear finite element, SCST.

\section{Introduction}

The main advantage of cable-tensioned and shaped space structures is their fast and economical construction and fabrication in special construction site. In order to develop more innovative construction techniques, extensive research has been carried out on the behavior characteristic of form finding for space structures by means of cable tensioning method. In general, because a space structure consists of many joints that are sensitive to stress and strain, and the characteristic of this joint effect to total behavior of structure, so it is necessary to handle carefully in design and construction. Shape formation test and theoretical analysis for the space structures were conducted with the small scaled test models that were consisted of only bolt type joint, bolt type joint with gusset plate, and ball type joint and steel members. Because the planar layouts are assembled at ground level with a singlelayer of top chords and pyramid units of web members, work efficiency and safety are increased. Some new types of cabletensioned and shaped space structure have been studied for the domes, arch, hypar shaped space structures (Kim et al. 20002008). In this study, specially for the three kinds of joint types in space structures, the experiments for the form finding have been performed with applying the cable tensioning to the cable located in the bottom chords, these results are compared with the results of the nonlinear analysis, and the characteristics of the behavior for each models are analyzed. As a result, the authors can suggest the possibility and feasibility of the form 
finding, and the behavior characteristic of joint for each space structures.

\section{Form Finding Principle of SCST Structure System}

The shape formation principle of cable tensioned and shaped space structures described in this paper is based on the mechanism condition and geometric compatibility condition. A mechanism condition means that a mechanism or near mechanism conditions (flexure only the top chords) must exist in its initial configuration, and that no mechanisms are allowed to exist in its final configuration. The mechanism condition of a cable-tensioned and shaped space structure in three-dimensional structure can be expressed by a general Maxwell's criterion.

$$
R-S+M=0 ; \quad \text { Where, } R=b-(3 j-r)
$$

Where, $R=$ degree of statical indeterminacy; $S=$ number of independent prestress states that exist; $M=$ number of independent mechanisms; $b=$ total number of members; $j=$ total number of joints; and $r=$ number of restraints on the structure. Using this criterion, a mechanism condition of a cable-tensioned and shaped space structure can be expressed as: $M>0(R<0$, $S=0)$ in its initial planar layout, and $M=0$ $(R \geq 0, S \geq 0)$ in its final space shape. The suitable basic structural type of the cable tensioned and shaped space structure is single-chorded space truss, it is called SCST structure. In the initial planar configuration for cable tensioning, it is the SCST condition, so it has the mechanisms or near mechanisms, for these reasons, SCST can be shaped easily with relatively small cable tensioning forces. Because the SCST structure is very weak structure, it can resist for deformation with only its weight, the friction of its joints, and flexural stiffness of the top chords. But after the cable tensioning and the self-locking process, the SCST can be a stable structure. Though the cable tensioning process may reduce the load resisting capacity, due to the existence of compressive pre-stress forces in some critical members after shape formation, the reduction in ultimate load capacity of cable-tensioned and shaped space structure could be improved by stiffening only a few critical members decided in analysis. And although the top chords are flexed during the cable tensioning, this bending is not serious for the safety of the structural behavior (Dehdashti 1996).

\section{Planar Layouts of Experimental Models}

General features of the small scaled test models for this study are as following; for the test model (A) as shown in Figure 1, the model shape is the circular type (diameter: $2,488.25 \mathrm{~mm}$ ), it is consisted of top chords of square hollow section (SHS) $13 \times 13 \times$ $1.5 \mathrm{~mm}$, web members of circular hollow section (CHS) $13 \times 2.5 \mathrm{~mm}$, bottom chords of CHS $13 \times 2.5 \mathrm{~mm}$. And the Poisson's ratio is 0.3 , yield stresses $\left(\sigma_{y}\right)$ are $450 \mathrm{MPa}$ for top chord, $440 \mathrm{MPa}$ for web chord, $440 \mathrm{MPa}$ for bottom chord, and Young's modulus (E) is $200 \mathrm{GPa}$. The top chords and webs are connected with the bolt type joint by gusset plate, the bottom of web members are welded joint as shown in (A) of Figure 4.

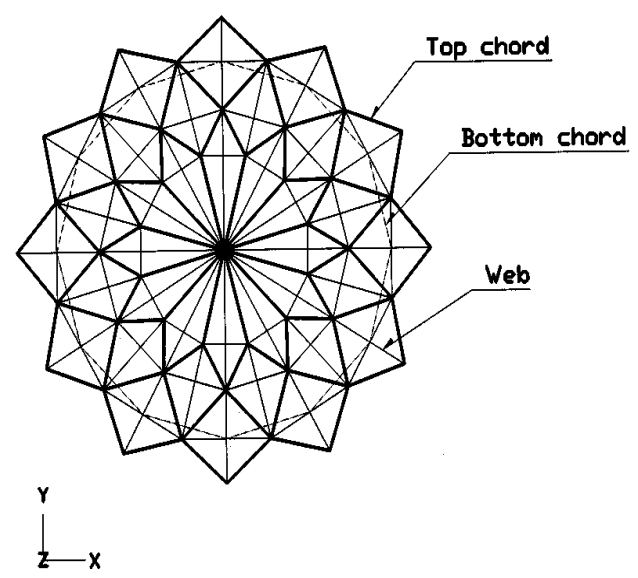

Fig. 1. Layout of test model (A). 
And the cable tensioning is applied to the direction of circumference along the bottom chord for finding the dome shaped test model. For the model (B) as shown in Figure 2, the test model is rectangular type, the top chords were made of SHS $13 \times 13 \times$ $1.8 \mathrm{~mm}$ steel tubes, while the web members were made of CHS $13 \times 2.0 \mathrm{~mm}$ steel tubes, the bottom chords were made of CHS $27 \times$ $8.75 \mathrm{~mm}$ steel tubes. The Young's modulus of the steel was $200 \mathrm{GPa}$, and Poisson's ratio was 0.3 . The top chords and web members were of nominal yield strength Grade 350 $\left(\sigma_{y}=350 \mathrm{MPa}\right)$ steel. In the test model (B), the connections of the top chords and web members are made of bolt type joint without gusset plate as shown in (B) of Figure 4 . And the cable tensioning is applied to the direction of diagonal for forming the hypar shaped test model. For the test model (C) as shown in Figure 3, all members are consisted with CHS $40 \times 4 \mathrm{~mm}$. The Young's modulus is taken as $136 \mathrm{GPa}$, Poisson's ratio is 0.3 and yield strength is $334 \mathrm{MPa}$. The joints of all of the members are connected with the ball type joint as shown in (C) of Figure 4. And the cable tensioning is then applied to the direction of middle line (bold line) to obtain the arch shaped test model.

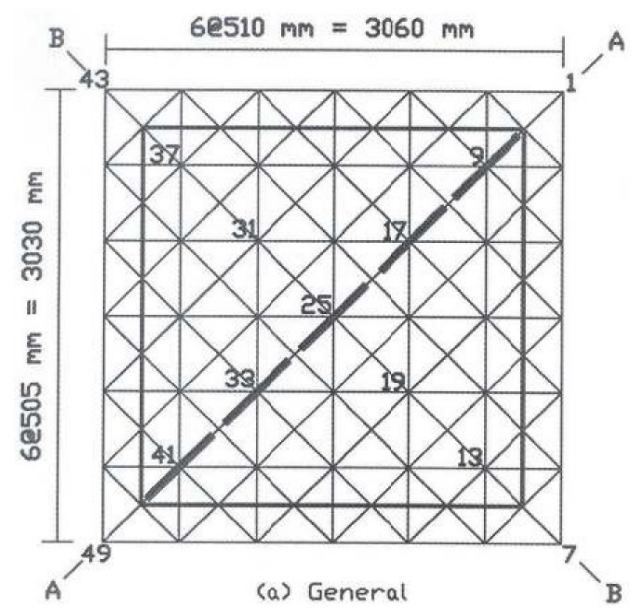

Fig. 2. Layout of test model (B).
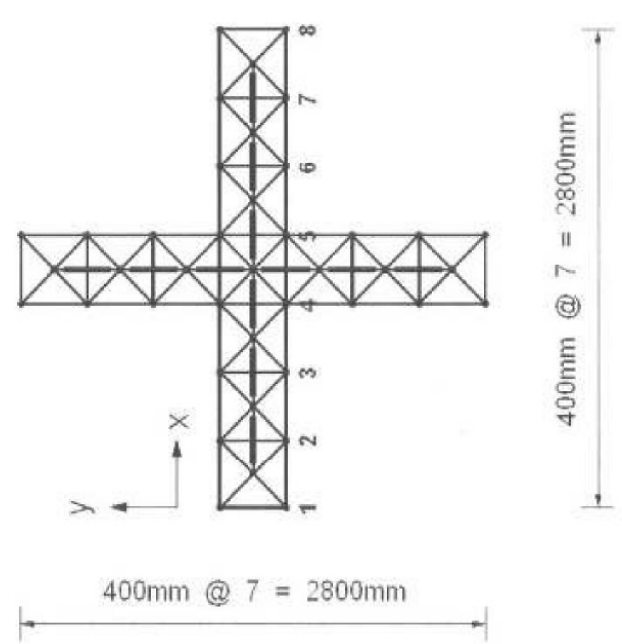

Fig. 3. Layout of test model (C).

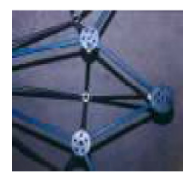

(A)

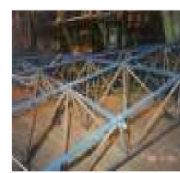

(B)

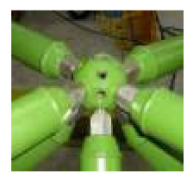

(C)
Fig. 4. Connection types of each models $(A, B, C)$.

\section{Analysis and Experiments for Form Finding}

A finite element simulation for the form finding must exactly represent the practical procedure of shaping. In such an analysis, the important point is how to model the mechanism for the closing of the gaps in the bottom chords. In the finite element analysis herein, the closing of the gaps in the bottom chord gaps were simulated by the element shortening caused by a negative temperature loads, and nonlinear finite element analysis has been performed with to analyze the behavior of test models. The MSC/NASTRAN and MIDAS are used to consider the large displacement effects in the nonlinearity of the structure behavior. The size of gap in bottom chord is closely related to desired shape of space structure. By loading the cable tensioning force to the test model, when the gaps of bottom 
chords were closed, space structure was formed into its required final shape. Consequently when the gaps were completely closed between the each joint of the bottom chord, i.e. there were no gaps in bottom chords, the cable tensioning process was completed.

The final deformed space structures are shown in Figures 5, 6, 7. The vertical displacement of the top joint is plotted in Figures 8, 9, 10. For the test model (A) of dome shaped space structure, the trend of form finding between the theory and experiment was shown in Figure 8. The good agreement between the theoretical results and experimental results was obtained from the central part of the test model, but the edge part is some difference compared with the central part. For the test model (B) of

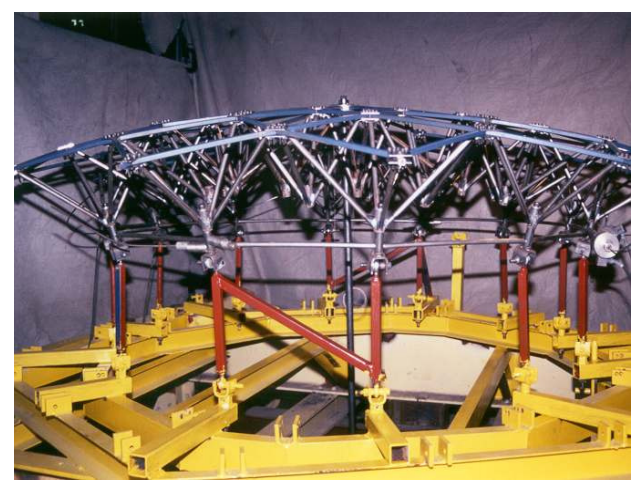

Fig. 5. Cable-tensioned space shape for test model (A).

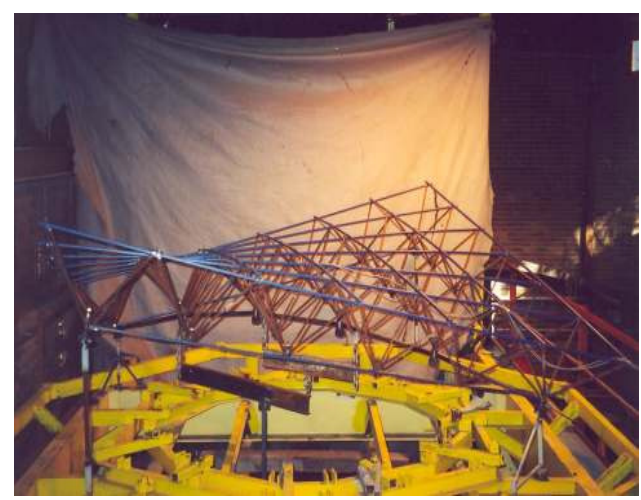

Fig. 6. Cable-tensioned space shape for test model (B).

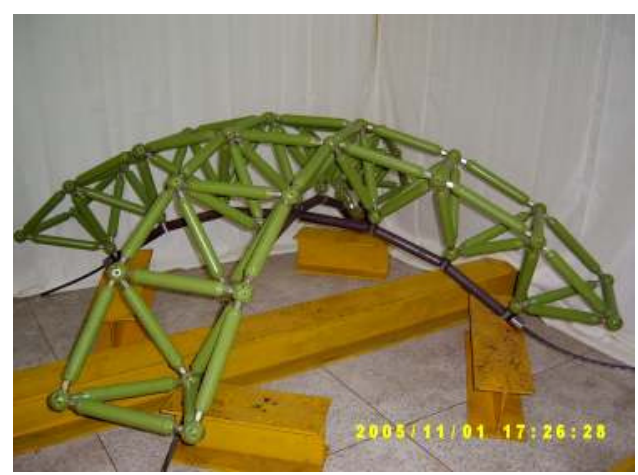

Fig. 7. Cable-tensioned space shape for test model (C).

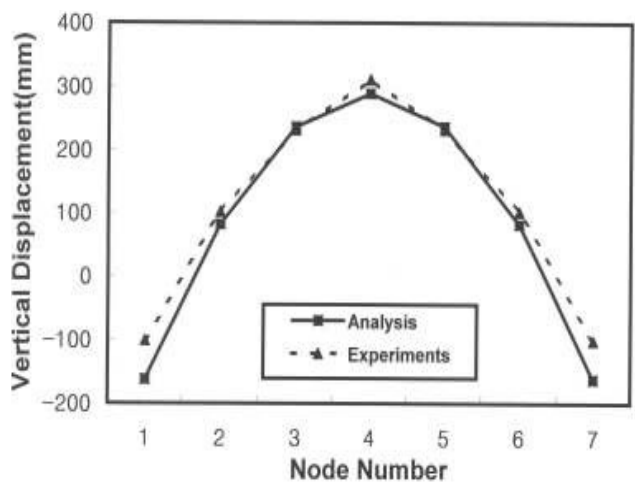

Fig. 8. Vertical displacement of top joints in model (A).

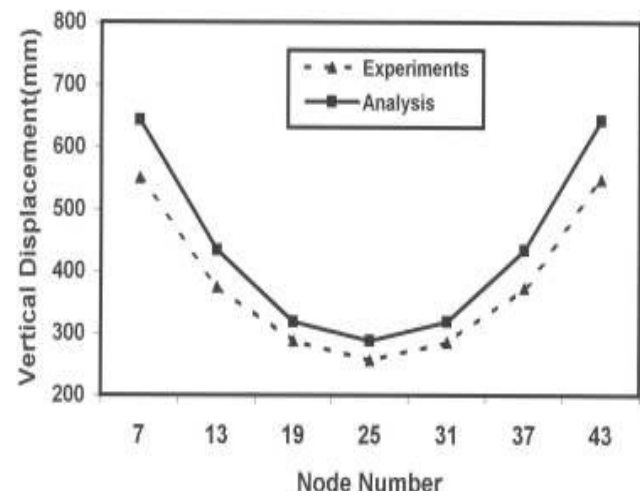

Fig. 9. Vertical displacement of B-B section for top joints in model (B).

hypar shaped space structure, in the case of $\mathrm{B}-\mathrm{B}$ section, the difference of form finding between the theory and experiment was shown in Figure 9, compared with other test models, this test model had compara- 


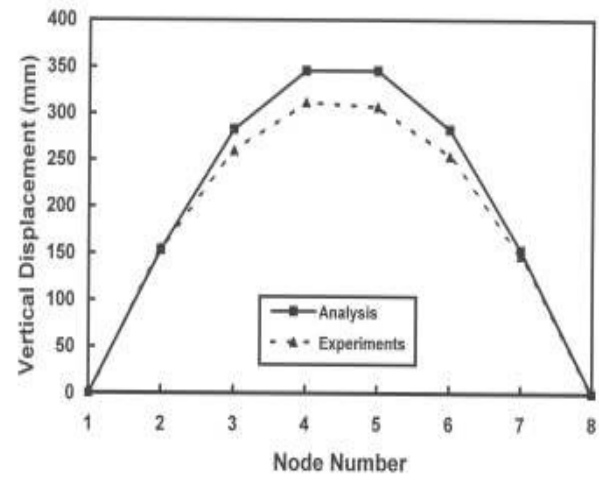

Fig. 10. Vertical displacement of top joints in model (C).

tive big discrepancies with theoretical and experimental results. It is an important reason that the joint is simple joint type, and the pyramidal units of the edges line have the lower chord, and the top chords are continuous at all test model, and it is different type compared with the other test models (A, C). For the test model (C) of arch shaped space structure, the behavior of shaping is almost similar with the test model (A), the difference for forming finding between the theory and experiment was shown in Figure 10. It is an almost good agreement between theory and experiment. In these experimental models, for the reasonable trends in the difference of the shaping between the theory and experiments according to each joint types, the reason for the some different trends of the good agreement is assumed that there are some characteristics of joint types. In this research the behavior characteristics of test models are similar to the results of author's previous researches for the many SCST structures. Through the experiments for the three types of SCST structures, within the yield limit of all the members, a planar layout can be deformed to desired space shape by means of cable tensioning forces. As a result, we can guess that the some differences between the theory and experiments in form finding for each test models are owing to the types of joints. Thus, for the design and construction in space structure, the behavior characteristic according to the joint type is more significant than those of any other member elements. Generally, in the form finding, some discrepancies between the theory and experiment exist due to the some geometric imperfections of test models, the rotations and slippage of joints, and the weakness of top chords stiffness owing to the bolt holes at joint in the test model. But as a mentioned above, nevertheless these imperfections affect the structural behavior of the form finding; most of these factors are not considered in detail for the finite element modeling. Consequently for the improvement of the efficiency in the finite element method for simulating the structural behavior of form finding of space structure involving mechanism or nearmechanism, further research is necessary.

\section{Conclusions}

Through the experiments and nonlinear finite element analysis for the form finding of three types jointed space structures, the following conclusions can be drawn.

The shaping formations for space structures with three types of joint are possible by cable tensioning, and in special site condition, the form finding of space structure by cable tensioning can be considered as a economic and time saved construction technique compared with the conventional techniques using a big crane or scaffold for erection.

A nonlinear finite element analysis method can be used for predicting the space shape and the cable tensioning forces in a form finding for every joint types of space structure. In form finding of SCST structures, the behavior of structures is mainly concerned with the connection type of joint. Due to the joint types, there are some discrepancies between the test model and theoretical model, so the behavior characteristic of the joint for the form finding needs further study.

\section{References}

Dehdashti, G. and Schmidt, L. C., Dome-Shaped Space Trusses Formed by Means of Posttensioning, Journal of Structural Engineering, ASCE, 122(10), 1240-1245, 1996. 
Kim, J. W. and Kwon, M. H., Shaping Formation and Behaviour Characteristic for SCST Structure by Cable-Tensioning, Journal of the Korea Society of Civil Engineers, 28(6A), 819-825, 2008.

Kim, J. W., Analysis and Test of Dome-Shaped Space Truss, Journal of the Korea Society of Civil Engineers, 20(1-A), 39-46, 2000.

Kim, J. W., Kim, T. S. and Lee, Y. H., Shape Formation of Space Structure with Ball Type Joint, The Joint International Conference on Computing and Decision Making in Civil and Building Engineering, Montreal (Canada), Paper No. IC-10, 2006.

Kim, J. W., Kwon, M. H. and Lee, Y. H., Influence of Removed Web Members in Shaping Formation for Hypar Space Truss, Journal of Ocean Engineering and Technology, 20(2), 16-21, 2006.

Kim, J. W., Kwon, M. H. and Rhew, H. J., Shaping Formation and Nonlinear Analysis of Mero Jointed Space Structure with Multi-directional Joint, Journal of the Architectural Institute of Korea, (121), 1118, 2005.

Kim, J. W., Doh, J. H. and Lee, Y. H., Behaviour Characteristic of Hypar Shaped Space Truss with Some Removed Members, Structure 2009 Congress, Vancouver (Canada), 2008.
Kim, J. W., Shape Creation and Ultimate Load Test of Space Truss by Means of Posttensioning, Journal of the Architectural Institute of Korea, 17(5), 51-57, 2001.

Kim, J. W. and Hao, J., Behaviour Characteristic of a Full-size Scale Pyramidal Space Truss Unit, KSCE Journal of Civil Engineering, 6(1), 3338, 2006.

Kim, J. W., Kim, J. J. and Rhew, H. J., Analysis and Experiment for the Formation and Ultimate Load Testing of a Hypar Space Truss, Journal of Constructional Steel Research, (62), 189-193, 2006.

Kim, J. W., Kim, J. J. and Rhew, H. J., Analysis and Experiment for Self-erected Hypar Space Truss, The Second International Conference on Structural Engineering, Mechanics and Computation, Cape Town (South Africa), 365-369, 2004.

Kim, J. W., Lee, Y. H. and Doh, J. H., Analysis and Test for Shaping Formation of Space Truss by Means of Cable-Tensioning, The 6th International Conference on Steel and Aluminium Structure, Oxford (UK), 2007. 